\title{
Complex Hemodynamic Insult in Combination with Wall Degeneration at the Apex of an Arterial Bifurcation Contributes to Generation of Nascent Aneurysms in a Canine Model
}

\author{
J. Wang, H.-Q. Tan, Y.-Q. Zhu, M.-H. Li, Z.-Z. Li, L. Yan, and Y.-S. Cheng
}

\begin{abstract}
BACKGROUND AND PURPOSE: The detailed mechanisms of cerebral aneurysm generation remain unclear. Our aim was to investigate whether specific hemodynamic insult in combination with arterial wall degeneration leads to the development of aneurysms in a canine model.

MATERIALS AND METHODS: New branch points in the common carotid artery were created in 18 dogs. Nine animals subsequently received elastase insult at the arterial bifurcation apex (elastase-treated bifurcation group); the control bifurcation group $(n=9)$ received saline, and 3 dogs received an elastase insult to both straight common carotid arteries (elastase-treated straight group). Angiographic and hemodynamic analysis was performed immediately and 12 and 24 weeks' postsurgery; histologic response was evaluated at 12 and 24 weeks.

RESULTS: Angiography revealed nascent aneurysms (mean, $3.2 \pm 0.4 \mathrm{~mm}$ ) at the arterial bifurcation apices in 5/9 models of the elastasetreated bifurcation group (versus 0 in the control bifurcation group and elastase-treated straight group) without any observed aneurysm rupture. Histologic analysis revealed internal elastic lamina discontinuity, elastic fiber disruption, a thinner muscular layer, reduced smooth-muscle cell proliferation, increased inflammatory cell (macrophage) infiltration, and expression of matrix metalloproteinase-2 and matrix metalloproteinase- 9 in the media of the elastase-treated bifurcation group compared with that in either the control bifurcation group or the elastase-treated straight group $(P<.001)$. Hemodynamic analysis after surgery indicated that the apex experienced extremely low wall shear stress and flow velocity and the highest relative and total pressure in the elastase-treated bifurcation group, while the values returned to normal after arterial wall remodelling.
\end{abstract}

CONCLUSIONS: In our study, combined hemodynamic insult and arterial wall degeneration at arterial bifurcations are required for the generation of aneurysms in a canine model.

ABBREVIATIONS: $C B G=$ control bifurcation group; $C C A=$ common carotid artery; EBG = elastase-treated bifurcation group; ESG = elastase-treated straight group; MAC387 = antimacrophage antibody; MMP = matrix metalloproteinase; SMC = smooth-muscle cell; WSS = wall shear stress; WSSG = wall shear stress gradient

B ifurcation aneurysms such as cerebral aneurysms have a high prevalence and can cause lethal hemorrhages ${ }^{1}$; however, the detailed mechanisms of aneurysm generation, growth, and rupture remain unclear. ${ }^{2}$

Received September 26, 2013; accepted after revision January 6, 2014. From the Department of Diagnostic and Interventional Radiology (J.W., H.-Q.T., Y.-Q.Z., M.-H.L., L.Y., Y.-S.C.), Shanghai Jiao Tong University Affiliated Sixth People's Hospital, Shanghai, China; and School of Mechanical Engineering (Z.-Z.L.), Shanghai Jiao Tong University, Shanghai, China.

This work was supported by grants from the National Natural Science Foundation of China (No. 81000652, 81370041), Shanghai Venus Program Funds (No.

11QA1405000), and the Med-Technological Crossing Funds (No. YG2011MS22).

We declare there is no conflict of interest in this study.

Please address correspondence to Yue-Qi Zhu, MD, and Ming-Hua Li, MD, No. 600, Yi Shan Rd, Shanghai, 200233, China; e-mail: zhuyueqi@hotmail.com and liminghuaradio@hotmail.com

-- Indicates open access to non-subscribers at www.ajnr.org

$D$ Indicates article with supplemental on-line video.

http://dx.doi.org/10.3174/ajnr.A3926
Advances in vascular biology, biomechanics, medical imaging, and computational methods provide an unprecedented opportunity to develop mathematic models to better understand aneurysm generation. Hemodynamic insult and arterial wall deficiency are both thought to play important roles in the generation of cerebral aneurysms because the muscular layer is absent within almost $80 \%$ of main cerebral arterial bifurcations, ${ }^{3,4}$ and cellular and molecular changes due to complicated wall shear stress (WSS) or wall shear stress gradient (WSSG) have been demonstrated in the bifurcated arterial wall at the aneurysmal zone. ${ }^{5}$ However, only a few satisfactory specific animal models that simulate the generation or growth of bifurcation aneurysms exist. ${ }^{6-8}$ We hypothesized that a combination of internal elastic lamina and media elastic fiber disruption and specific hemodynamic flow action may promote the generation and development of aneurysms. Therefore, we designed a canine common carotid artery (CCA) Y-type bifurcation model, with and without internal elastic lamina and media elastic fiber insult, to 
dynamically observe aneurysm growth at the angiographic and histopathologic levels.

\section{MATERIALS AND METHODS \\ Animals}

All protocols used in this study were approved by the Animal Research Committee of our institution and were conducted in accordance with the guidelines of the International Council on Animal Care. Twenty-four 8-month-old beagles of both sexes, weighing 12-20 kg, were used (Agronomic School of Shanghai Jiaotong University, License: SCXK2007-0004).

\section{Animal Surgery Model}

Arterial bifurcations with a new branch point were surgically created in the CCA of 18 dogs by using the method described by Meng et $\mathrm{al}^{7}$ and then were divided into an elastase-treated bifurcation group (EBG, $n=9$ ) and a control bifurcation group (CBG, $n=9)$, with 6 female and 3 male dogs in each group. Elastase (3.0 $\mathrm{U} / \mu \mathrm{L}$ ) was applied for 15 minutes to the apex of the reconstructed arterial bifurcation (approximately $4 \times 4 \mathrm{~mm}^{2}$ ) in 9 animals in the EBG by dripping it from a curved blunt syringe needle into a trapezoidal plastic tube, to prevent its flowing onto other portions of the arteries; the same volume of saline was applied in 9 animals as controls by using the method identical to that in the elastase model in the CBG. Before elastase or saline was applied, as much of the tunica adventitia at the apex as possible was removed to facilitate the penetration. Meanwhile, 3 dogs with both straight CCAs surgically exposed received elastase insult as an elastasetreated straight group (ESG). The peak velocity of blood flow in the feeding artery at the systolic phase was measured before and after reconstruction surgery by using Doppler ultrasound.

\section{Angiographic Analysis}

Angiography was performed immediately and 12 and 24 weeks after surgery. Immediate angiography, including 2D-DSA (anteroposterior and lateral positions) and 3D-DSA reconstructions, was performed to assess morphologic alterations at the apex of the reconstructed arterial bifurcation. If an aneurysm-like bubble was observed, its neck and maximum body width were measured by using the best $3 \mathrm{D}$-DSA projection, and parent artery diameter and the angulation between the parent arteries were determined. We defined an aneurysm formation only if its maximum body width exceeded half of the diameter of the adjacent artery. Ultrasonography was used as a convenient method to monitor the status of the aneurysms at 4 and 8 weeks, check whether aneurysms were generated, determine the size of the aneurysms, and confirm whether the reconstructed arterial bifurcations were patent.

\section{Computational Fluid Dynamics Analysis}

Computational fluid dynamics analysis was performed immediately and 12 and 24 weeks after surgery. With in vivo 3D-DSA rotational angiography (syngo Axiom-Artis; Siemens, Erlangen, Germany), the 3D lumen of the bifurcation was reconstructed and, together with the measured flow rates in the vessel branches, served as the boundary conditions for computational fluid dynamics simulations (Mimics 10.0; Materialise, Leuven, Belgium) to generate an advanced tetrahedral mesh inside the converted 3D surface. The average mesh size was approximately 153,565 U. The 3D computational fluid dynamics solutions were defined under steady-state (average peak Reynolds number $=625$ ), assuming a Newtonian fluid $(0.0035 \mathrm{~Pa} \times \mathrm{s})$ and incompressible flow by using a rigid-wall model (CFDesign AVL-Fire, Version 2008, AVL List $\mathrm{GmbH}$, Graz, Austria). WSS, velocity field, streamline, surface relative pressure field, and total pressure field were analyzed. The total pressure was defined as the total flow pressure during isentropic stagnation heating, while the relative pressure was defined as the pressure calculated on the basis of the local atmospheric pressure. In this study, the local atmospheric pressure was chosen as $10^{5} \mathrm{~Pa}$. The WSSG was calculated. The gradient normal to the streamline was negligible in comparison. Meanwhile, a quantitative analysis on the WSS and WSSG of the bifurcation models in the EBG was also performed.

\section{Histologic Examination}

Reconstructed arterial bifurcations were harvested at $12(n=6)$ and 24 weeks $(n=3)$ in the EBG and CBG, while straight-vessel samples $(n=6)$ were harvested at 24 weeks after surgery. CCA samples from the pilot study and surgical models were stained with hematoxylin-eosin, Masson trichrome, and elastic fiber stains (Verhoeff van Gieson/Orcein methods). Serial longitudinal sections of the reconstructed arterial bifurcations were obtained to assess tissue changes in relationship to the consistent hemodynamic action. CCA samples were immunostained by using proliferating cell nuclear antigen, smooth-muscle $\alpha$-actin, CD45, antimacrophage antibody (MAC387), and matrix metalloproteinase-2 (MMP-2) and matrix metalloproteinase-9 (MMP-9) antibodies. Double immunofluorescent staining was performed by using anti-CD45 for pan-leukocytes and anti-MAC387 for macrophages to assess whether macrophages were included in leukocytes. The smooth-muscle cell (SMC) proliferation rate (percentage of proliferating cell nuclear antigen-positive SMCs in the media) and elastic layer and $\alpha$-actin-positive SMC layer thickness were measured. The inflammatory cell infiltration index was defined as the percentage of CD45-positive cells in the media of the arterial bifurcation apices. The MMP-2 and MMP-9 expression indexes were defined as the percentage of MMP-2/9-positive area in the media of the arterial bifurcation apices.

Statistical analysis of the histopathologic images was performed with Image-Pro Plus, Version 6.0 software (Media Cybernetics, Rockville, Maryland) in at least 20 randomly selected highpower $(\times 400)$ tubulointerstitial fields from each section.

\section{Statistical Analysis}

GraphPad Prism 5.0 software (GraphPad Software, San Diego, California) was used for statistical analysis. Data were expressed as the mean \pm SD for continuous variables and as counts or proportions (percentage) for categoric variables. The Fisher exact test was used to compare categoric data. Grouped $t$ tests were used to compare the proliferating cell nuclear antigen proliferation index, elastic layer and media $\alpha$-actin-positive layer thickness, inflammatory cell infiltration index, and MMP-2 and MMP-9 indexes. All tests were 2-tailed, and statistical significance was defined as $P<.05$. 

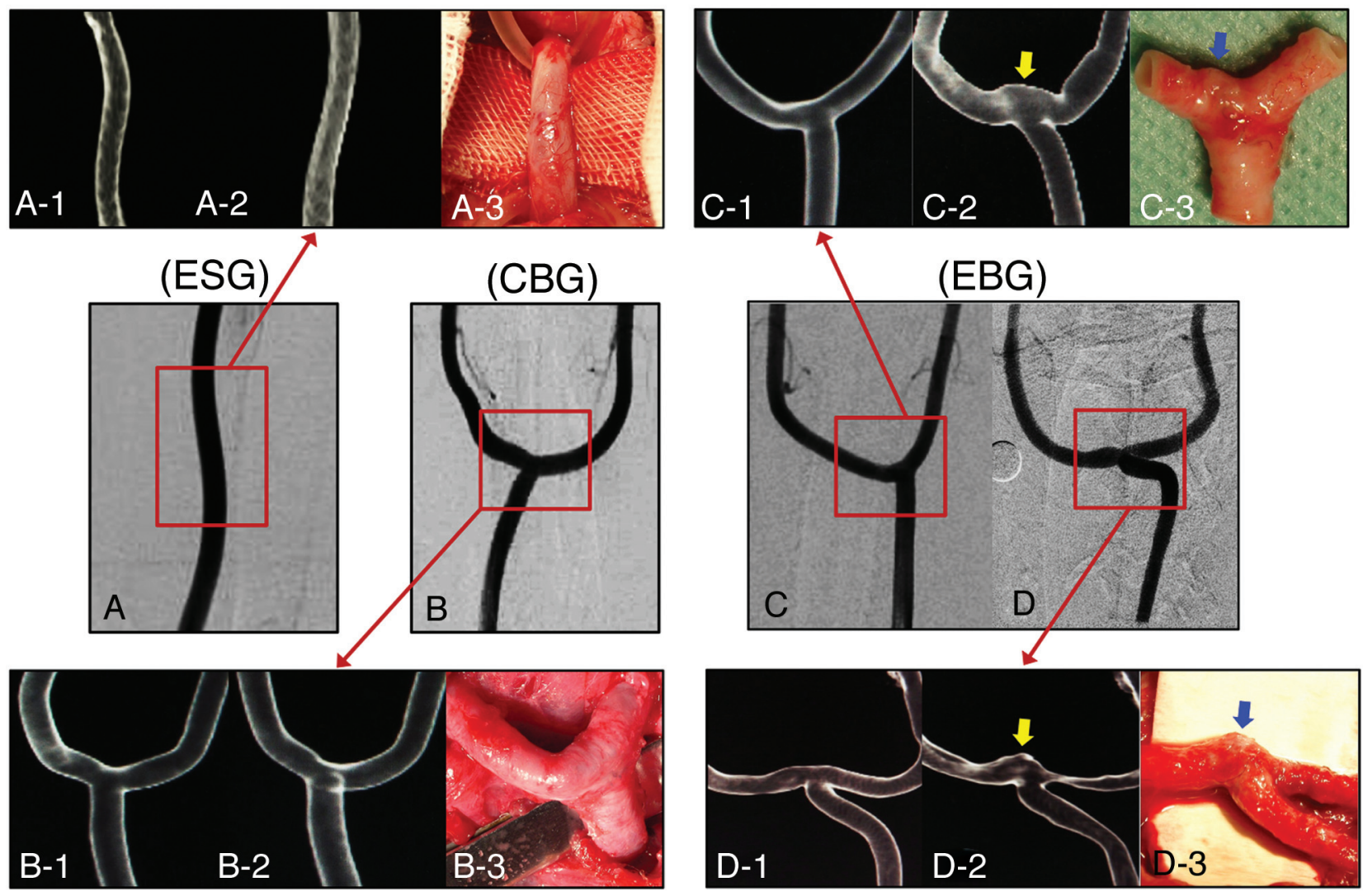

FIG 1. 2D-DSA and 3D-DSA images from immediately after surgery. 3D-DSA follow-up and gross dissection in the ESG (A), CBG (B), and EBG (C and $D)$. Yellow and blue arrows indicate nascent aneurysms.

Summary of the information of dog models in the ESG, CBG, and EBG ${ }^{a}$

\begin{tabular}{|c|c|c|c|c|c|c|c|c|c|c|}
\hline \multirow{3}{*}{$\begin{array}{l}\text { Group } \\
\text { (No.) }\end{array}$} & \multirow{3}{*}{$\begin{array}{l}\text { Sex } \\
(\mathrm{F})\end{array}$} & \multirow[b]{3}{*}{ Weight (Kg) } & \multirow{3}{*}{$\begin{array}{l}\text { Surgical } \\
\text { Results }\end{array}$} & \multirow{2}{*}{\multicolumn{2}{|c|}{$\begin{array}{c}\text { Blood Flow Velocity } \\
(\mathrm{cm} / \mathrm{s})\end{array}$}} & \multirow{3}{*}{$\begin{array}{l}\text { PA Diameter } \\
(\mathrm{mm})\end{array}$} & \multirow{3}{*}{$\begin{array}{l}\text { PA Angulation } \\
\text { (Degrees) }\end{array}$} & \multicolumn{3}{|c|}{ Aneurysm Status } \\
\hline & & & & & & & & \multirow{2}{*}{$\begin{array}{c}\text { Sex } \\
\text { (No./Female) }\end{array}$} & \multirow{2}{*}{$\begin{array}{l}\text { Neck } \\
(\mathrm{mm})\end{array}$} & \multirow{2}{*}{$\begin{array}{l}\text { Body } \\
(\mathrm{mm})\end{array}$} \\
\hline & & & & Before & After & & & & & \\
\hline ESG (3) & 2 & $14.8 \pm 1.4$ & Success & $82.4 \pm 6.3$ & - & $4.1 \pm 0.2$ & - & 0 & - & - \\
\hline CBG (9) & 6 & $14.7 \pm 2.4$ & Success & $84.9 \pm 6.5$ & $126.2 \pm 12.5^{b}$ & $4.3 \pm 0.4$ & $112.9 \pm 36.1$ & 0 & - & - \\
\hline EBG (9) & 6 & $14.9 \pm 1.7$ & Success & $85.7 \pm 8.7$ & $131.4 \pm 13.9^{b}$ & $4.3 \pm 0.4$ & $120.3 \pm 44.2$ & $5 / 4$ & $6.7 \pm 2.3$ & $3.2 \pm 0.4$ \\
\hline
\end{tabular}

Note:-F indicates female; PA, parent artery.

${ }^{a}$ Data are expressed as the mean \pm SD for continuous variables and as counts for categoric variables.

${ }^{\mathrm{b}}$ Statistical significance before and after surgery within each group $(P<.05)$.

\section{RESULTS}

\section{Animal Surgery Model and Angiographic Findings}

The arterial bifurcation model was successfully reconstructed in all animals. Ultrasonography or angiography revealed no stenosis at the anastomoses or arterial occlusion postsurgery or during follow-up in any animal. Angiography indicated that the vessel lumen at the arterial bifurcation apex herniated to form an aneurysm in 5/9 animals, with 4 aneurysms in female dogs (66.7\%) and 1 aneurysm in a male $\operatorname{dog}(33.3 \% ; P>.05)$ from the elastasetreated group, and 2D-DSA and 3D-DSA revealed that bubbles were located at the apices, with a tent shape (mean sac diameter, $3.2 \pm 0.4 \mathrm{~mm}$; mean neck diameter, $6.7 \pm 2.3 \mathrm{~mm}$; Fig 1 ). However, the aneurysms were still at their unruptured stage until 24 weeks' follow-up, with only slight saccular enlargement, compared with 12 weeks in the EBG $(3.5 \pm 0.3 \mathrm{~mm}$ versus $3.0 \pm 0.2$ $\mathrm{mm} ; P=.076)$. Ultrasonography monitoring revealed that all of the aneurysms were detectable at 4 weeks. No animal in the control group displayed any morphologic changes on angiography at
12 weeks. The mean parent artery diameter and angulation were $4.3 \pm 0.4 \mathrm{~mm}$ and $112.9 \pm 36.1^{\circ}$ in the CBG and $4.3 \pm 0.4 \mathrm{~mm}$ and $120.3 \pm 44.2^{\circ}$ in the EBG, respectively (Table). However, the average parent artery angulation of the $5 / 9$ animals with aneurysms in the EBG was $146.8 \pm 40.84^{\circ}$ compared with $87.25 \pm 18.87^{\circ}$ in models with no aneurysms $(P=.032)$.

\section{Arterial Wall Remodelling in Histology}

In the 5 arterial bifurcation samples with aneurysms on angiography, biopsy confirmed that the entire vessel wall was thinned with a semitransparent appearance and visible blood flow underneath, which enabled herniation and aneurysm formation at the arterial bifurcation apices (On-line Video).

Hematoxylin-eosin and Masson trichrome staining revealed thinning of media at the bifurcation, a reduced numbers of SMCs, and loss of fibronectin in the elastase-treated group. Elastic fiber staining revealed internal elastic lamina discontinuity and elastic fiber disruption/insult in all elastase-treated animals in the ESG 

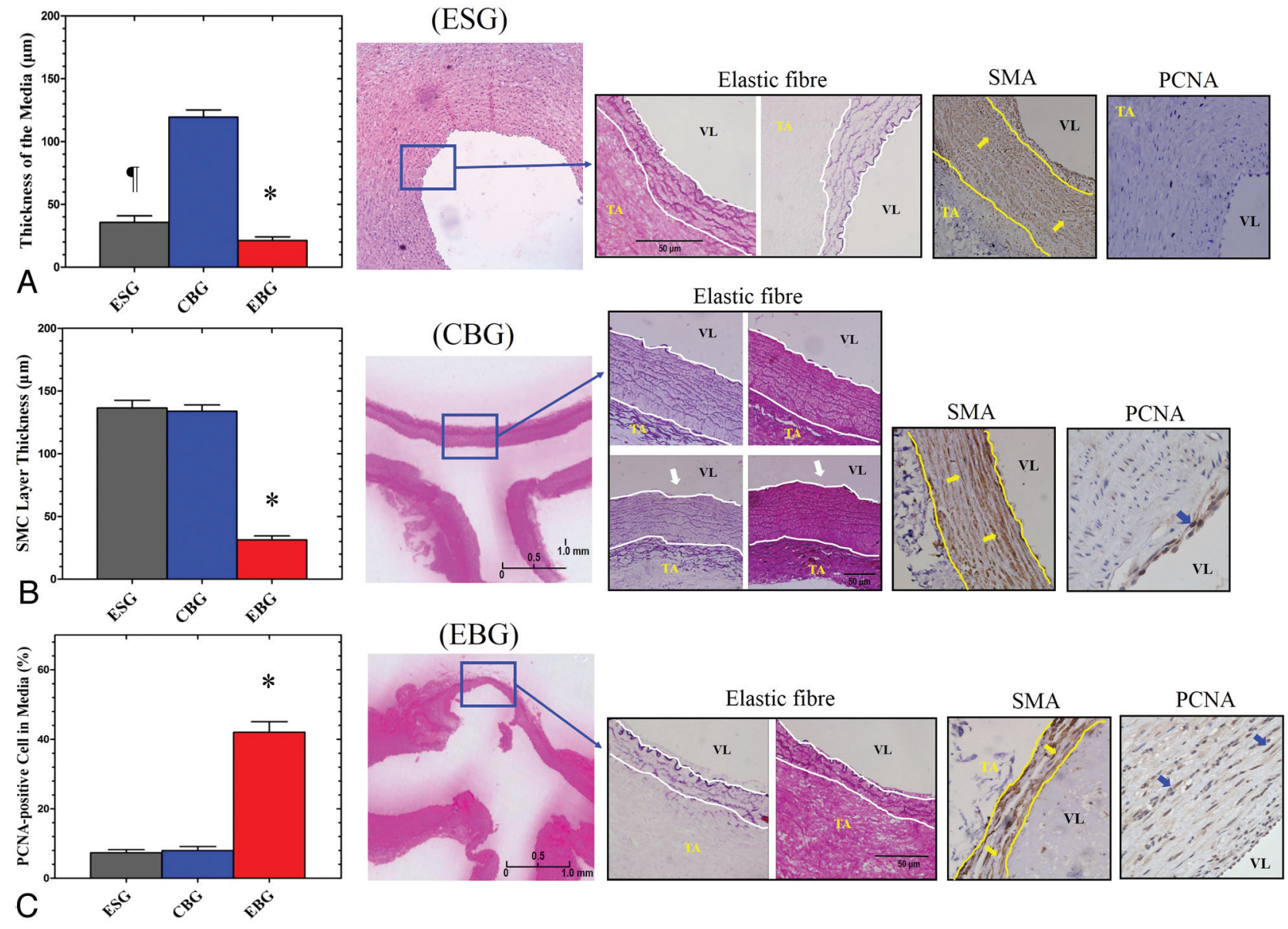

FIG 2. A, Specific elastic fiber staining, which shows the thickness of the media (white lines; $\times 100$ ), indicated significant thinner of the media in the ESG than in the CBG and EBG.). Internal elastic lamina discontinuity is indicated by white arrows. $B$, Immunostaining of smooth-muscle $\alpha$-actin (yellow arrows; smooth-muscle actin layer, yellow lines; $\times 100)$ and proliferating cell nuclear antigen $(C$, blue arrows) in the media of the straight artery and arterial bifurcation apices in the ESG, CBG, and EBG $(P<.001$ versus ESG and CBG; $\times 400)$. The asterisk indicates $P<.05$ when the ESG, CBG, and EBG were compared. The flag indicates $P<.05$ when the ESG and CBG were compared. VL indicates vessel lumen; TA, tunica adventitia.

and EBG; only 3 animals from the CBG displayed intima layer disruption with mild internal elastic lamina discontinuity at the arterial bifurcation apices. Elastase treatment reduced the media elastic layer thickness in the EBG $(21.2 \pm 15.3 \mu \mathrm{m})$ and ESG $(71.28 \pm 19.47 \mu \mathrm{m})$ compared with that in the CBG $(119.4 \pm 29.9$ $\mu \mathrm{m} ; P<.001$; Fig 2).

Immunohistochemical staining revealed increased numbers of proliferating cell nuclear antigen-positive SMCs in the media of elastase-treated arterial bifurcation apices in the EBG (42.0 \pm $15.7 \%$ versus $7.3 \pm 3.8 \%$ in ESG and $8.0 \pm 6.3 \%$ in the $\mathrm{CBG} ; P<$ .001 ; Fig 2) The number of smooth-muscle $\alpha$-actin-positive SMCs also decreased, and the SMC layer was thinner at elastasetreated arterial bifurcation apices in the EBG $(31.3 \pm 16.7 \mu \mathrm{m}$ versus $136.5 \pm 25.5 \mu \mathrm{m}$ in the ESG and $133.9 \pm 26.1 \mu \mathrm{m}$ in the CBG; $P<.001$; Fig 2). The inflammatory cell infiltration index in the media of the aneurysm wall was $38.4 \pm 10.6 \%$ in the EBG (versus $5.1 \pm 2.1 \%$ in the ESG and $2.9 \pm 2.4 \%$ in the CBG; $P<$ $.001)$. Macrophage staining showed macrophage infiltration into the aneurysmal wall with a distribution similar to that of leukocytes. Furthermore, double staining with anti-CD45 and antiMAC387 revealed that some leukocytes in the media of the aneurysm wall in this model were macrophages. The MMP-2 and
MMP-9 expression indexes in the media of the aneurysm wall were $21.0 \pm 8.7 \%$ (versus $1.2 \pm 1.4 \%$ in the ESG and $0.8 \pm 1.2 \%$ in the $\mathrm{CBG} ; P<.001$ ) and $13.6 \pm 5.6 \%$ (versus $0.9 \pm 0.8 \%$ in the ESG and $0.4 \pm 0.6 \%$ in the CBG; $P<.001$ ), respectively (Fig 3 ).

\section{Computational Fluid Dynamics Analysis}

Flow rates in the feeding artery of the de novo bifurcation increased from $85.3 \pm 7.5 \mathrm{~cm} / \mathrm{s}$ to $128.8 \pm 13.1 \mathrm{~cm} / \mathrm{s}$ (approximately $51 \%$ ) due to ligation of the contralateral CCA. Computational fluid dynamics analysis immediately after surgery revealed that the feeding artery wall experienced increased WSS in the CBG and EBG, whereas the bifurcation periapical regions were exposed to a more complex hemodynamic environment. The arterial bifurcation apex had extremely low WSS and flow velocity, and from the apex to the bilateral vessel wall, the WSS and flow velocity increased to a maximum and then decreased further downstream to a level similar to that of the feeding artery. This acceleration and deceleration created a WSSG-positive region (before maximum WSS) and -negative region (after maximum WSS). The apex also experienced the highest relative pressure and total pressure, which decreased to normal values toward both branch arms. 


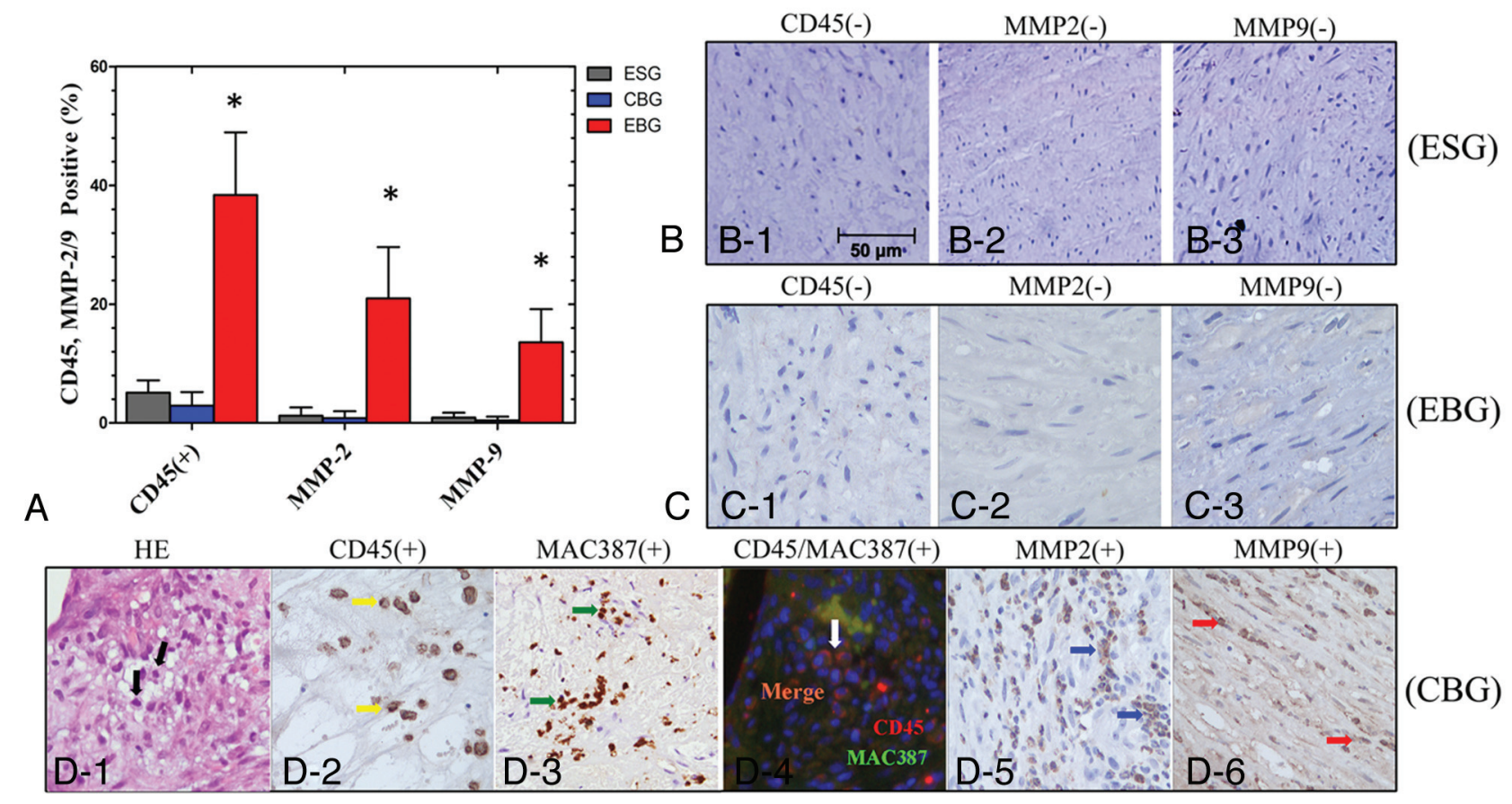

D

FIG 3. Immunohistochemical staining and quantification analysis $(A)$ of infiltrating inflammatory cells (CD45+/MAC $387+$ ) and MMP- 2 and -9 expression in the media of straight vessels and at the arterial bifurcation apices ( $\times 400)$ in the ESG $(B), C B G(C)$, and EBG $(D)$. The asterisk indicates $P<.05$ when the ESG, CBG, and EBG were compared.
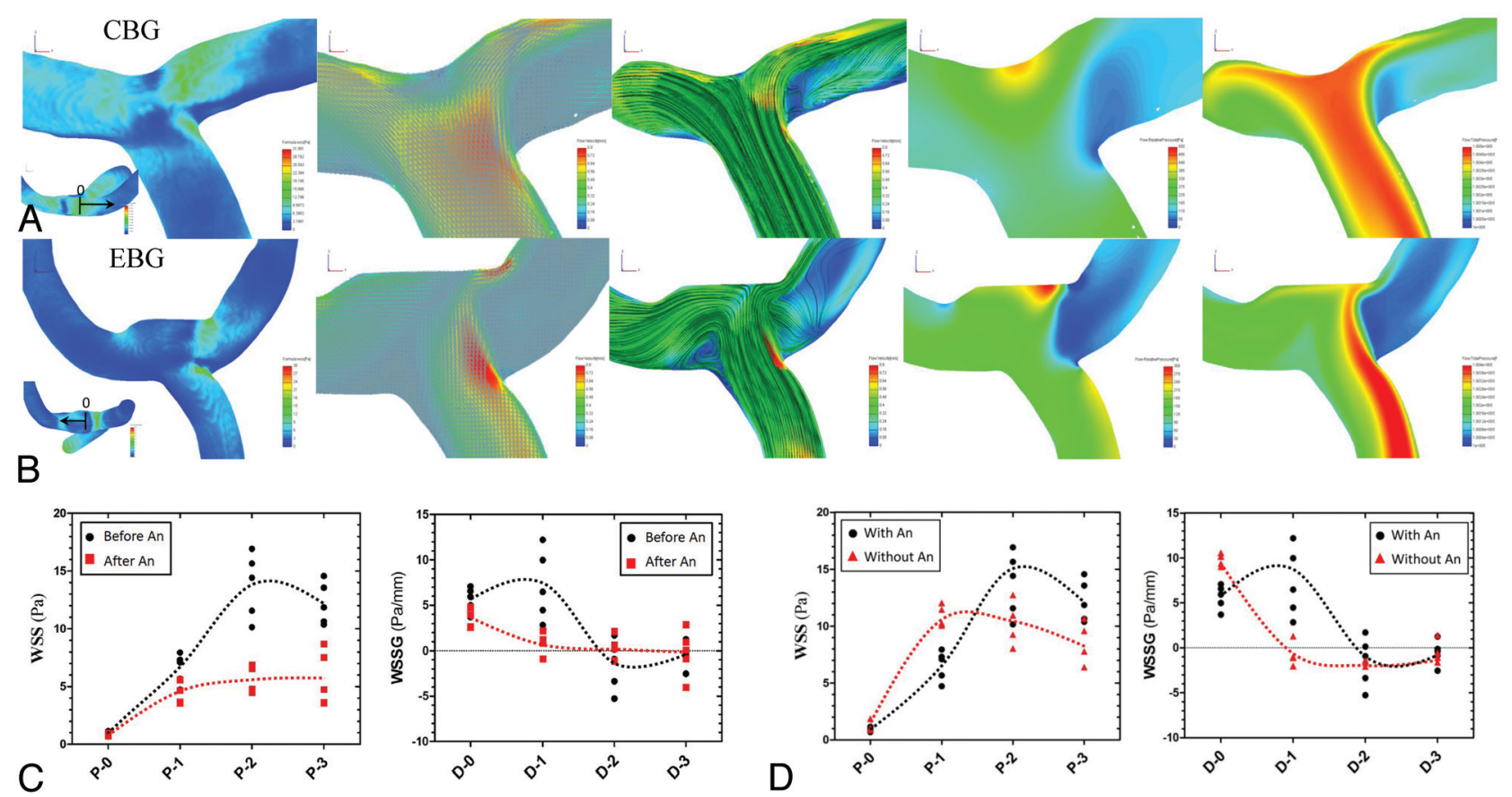

FIG 4. Computational fluid dynamics analysis at 12 weeks of follow-up in the CBG $(A)$ and EBG $(B)$. A comparison of the changes of WSS and WSSG values before and after aneurysm formation in models with an aneurysm in the EBG $(C)$ and the WSS and WSSG values immediately after surgery in models with large and small bifurcation angles in the EBG (D). A dotted line indicates the varying trends of WSS and WSSG in each subgroup.

Hemodynamic parameters including WSS, blood velocity, streamline field, surface relative pressure field, and total pressure field were similar immediately after surgery and 24 weeks after surgery in the ESG, CBG, and the 4/9 animals in the EBG without morphologic changes, as illustrated in Fig $4 A$. In the 5/9 elastasetreated animals with aneurysms in the EBG, quantitative analysis of the WSS and WSSG values, including at the apex and equidistant points $(1,2$, and $3 \mathrm{~mm})$ on one of the affected sides, indicated 
an obvious decrease from immediately after surgery to 24 weeks after surgery (WSS: $13.74 \pm 2.82$ versus $5.48 \pm 1.14 \mathrm{~Pa}$ at the 2-mm point, $P<.01$; WSSG: $7.19 \pm 3.87$ versus $0.85 \pm 1.12$ $\mathrm{Pa} / \mathrm{mm}$ at the $1-\mathrm{mm}$ point, $P=.01$ ), in a manner similar to that in the distal outflow vessel, due to arterial remodeling leading to the formation of an aneurysm sac (Fig $4 B,-C$ ). In these animals, the flow velocity, relative pressure, and total pressure at the apex also decreased during follow-up. Further quantitative analysis of the WSS and WSSG values immediately after surgery in the EBG also indicated a higher WSS (often $2 \mathrm{~mm}$ besides the apex) and WSSG (often $0-2 \mathrm{~mm}$ besides the apex) insult to the arterial wall in models with an aneurysm than in those without one (Fig 4D). The arterial walls subjected to high WSS and WSSG values were confirmed to show remodeling by both angiographic and histologic observations. Considering that models that developed an aneurysm often had larger bifurcation angles, the arterial wall adjacent to the apex would be subjected to higher WSS and WSSG values.

\section{DISCUSSION}

Further knowledge of the pathologic mechanisms underlying aneurysm generation or growth may provide the opportunity to develop mathematic models to better understand the progression of vascular diseases. Until recently, animal models have aimed to evaluate the underlying mechanisms of aneurysm formation by simulating possible aneurysm-inducing factors, such as altered hemodynamics, and have compared the morphologic or histopathologic changes with those in humans. ${ }^{6-8,9}$ In 2007, Meng et $\mathrm{al}^{7}$ surgically created a new bifurcation site in the canine carotid artery and reported a correlation between high WSS or WSSG and histologic insult; however, arterial wall aneurysms did not develop, and further study is required to confirm whether the observed histologic changes induced aneurysm formation. In 2008, Gao et $\mathrm{al}^{6}$ induced nascent cerebral aneurysms by increasing hemodynamic stress via bilateral ligation of rabbit carotid arteries. Increased hemodynamic stress triggered nascent aneurysm formation and correlated with increased blood flow in the basilar artery; however, the histologic changes could not be confirmed by traditional intra-arterial angiograms or other radiologic methods due to the tenuous blood vessel of the rabbit. Subsequent studies have reported that complex hemodynamics at bifurcation sites can induce molecular changes in a manner similar to those in human aneurysmal wall changes, including decreased expression of endothelial nitric oxide synthase, CD68 deficiency, and up-regulation of MMP-2, MMP-9, inducible NO synthase, and interleukin- $1 \beta$, which is restricted to the high WSS and high WSSG areas, ${ }^{5,10,11}$ suggesting that altered hemodynamics act at a molecular level to promote aneurysm formation.

Besides constant blood flow action, many studies have supported the suggestion that factors leading to deterioration of the arterial wall structure are the most important step in cerebral aneurysm development. ${ }^{12}$ The apex of cerebral vasculature bifurcations differs from that in most large arteries because the median muscular layer is usually absent. In addition, cerebral vessels exhibit a fragile and/or absent external elastic lamina and are surrounded by CSF, thus rendering them susceptible to injury from mechanical forces. ${ }^{3,4,12}$ These observations have led to the hy- pothesis that structural wall abnormalities and defective collagen and elastin are the major culprits in aneurysm initiation. ${ }^{13}$ In 2009, Nuki et $\mathrm{al}^{8}$ reported a novel cerebral aneurysm model by inducing hypertension and the degeneration of elastic lamina in mice and found that cerebral aneurysm generation was closely related to MMP activation ${ }^{8}$; however, the aneurysm site was not controllable.

In this study, we investigated the role of arterial bifurcation hemodynamic simulation combined with arterial wall degeneration and blood flow acceleration in a canine model of aneurysm generation. Specifically, we induced arterial wall degeneration via internal elastic lamina and media elastic fiber insult by using elastase to induce muscular layer degeneration after blood flow action. Using the method of Meng et al, ${ }^{7}$ we reconstructed bilateral CCA bifurcation apices to simulate the hemodynamic conditions and increased blood flow by unilateral CCA ligation. Because inner arterial wall elastase incubation led to a high rate of intra-arterial thrombus occurrence in preliminary experiments, we used an external wall incubation method, which selectively disrupted the internal elastic lamina and elastic fibers to simulate arterial wall degeneration. The removal of as much tunica adventitia as possible to facilitate elastase penetration was key.

Despite the fact that the blood flow pressure and velocity are similar in small animals and humans, the vessel wall structures, especially the arterial muscle layer, are very different. Consequently, aneurysms induced in small animals may not be reproducible in large animals. Here, we used a canine model because canine vessels behave more like human vessels. ${ }^{14}$ The long canine CCA $(10-12 \mathrm{~cm})$ provides sufficient length to construct an arterial bifurcation model. The caliber of the canine CCA and human ICA are comparable, with a constant diameter of approximately 4 $\mathrm{mm}$. Moreover, the cerebral blood supply in dogs is delivered almost entirely through the vertebrobasilar system, with anastomoses between each network of the ICA, ${ }^{15}$ which helps prevent death when the bilateral CCA is temporarily occluded. Additionally, the number of cells, their composition, and the accompanying proteoglycan matrices in the canine CCA are essentially the same as in the human CCA, ${ }^{16}$ indicating that canine models may accurately predict the human biologic response to aneurysm-inducing factors.

During 24-weeks' follow-up, aneurysms were successfully induced in 5/9 elastase-treated animals in the EBG and were confirmed by both angiography and histopathology. We hypothesize that arterial wall deterioration, combined with distinct hemodynamics, effectively results in aneurysm initiation. The weakened arterial wall at the apex may adapt or respond to sustained alterations in blood flow, blood pressure, or axial stretch. Aneurysm generation or enlargement is a type of arterial wall remodelling, which may potentially involve increased inflammatory cell infiltration, increased MMP activity, extracellular matrix protein synthesis, and SMC apoptosis, to counteract the mechanical forces induced by altered blood flow. ${ }^{17}$ Inflammatory cell infiltration was detected in the media of the aneurysm walls by pan-leukocyte staining by using an anti-CD45 and MAC387 antibody, consistent with observations in animal and human intracranial aneurysms, ${ }^{2,18}$ indicating that macro- 
phage infiltration plays a critical role in the formation of the aneurysms observed in this model, especially during the early stages of aneurysmal formation. MMP-2 and MMP-9 were also expressed in the walls of the aneurysms, both of which possess gelatinase and collagenase activity and can degenerate the important extracellular matrix components in the walls of aneurysms, such as elastin and collagen type IV. ${ }^{19}$ Taken together, these results indicate that MMP may be released from the infiltrating macrophages and may play an important role in the progression of aneurysms.

The aneurysms had a wide neck, mainly determined by the size of the elastase-incubated area, and a small body; therefore, they can only be termed "nascent aneurysms." However, these nascent aneurysms had histologic characteristics similar to those in human aneurysms including the following: 1) internal elastic lamina discontinuity with a reduced media elastic layer and SMC layer thickness; 2) degeneration, thinning, or interruption of the media SMC layer; and 3) significantly reduced SMC proliferation. Another feature of this model was that no thrombus formation was revealed in the aneurysm sac at angiography or during the histologic examination. This suggests that the elastase insult to the adventitia of the bifurcation apex kept the endothelial cell layer intact, to prevent thrombosis and platelet aggregation in response to expression of CD39 and secretion of nitric oxide and prostacyclin. ${ }^{20}$

Blood flow is generally considered an important modulator of normal artery enlargement in response to increased flow to maintain normal shear stress. ${ }^{21}$ In this study, despite all models having simulated elastic fiber insults (arterial wall deterioration), nascent aneurysms were generated in only 5 of 9 models. We hypothesize that the bifurcation angle is an important factor because $\mathrm{T}$-shaped bifurcations $\left(146.8 \pm 40.84^{\circ}\right)$ were more likely to induce the generation of aneurysms than Y-shaped bifurcations (87.25 \pm $\left.18.87^{\circ}\right) .{ }^{22}$ Small bifurcation angles are associated with high blood flow velocities and dispersed vortex intensities, while increased bifurcation angles result in more concentrated vortex intensities next to the apex and turbulent/disturbed laminar flow, which generates various mechanical stimuli, such as WSS and WSSG. $^{23,24}$ The quantitative analysis of the WSS and WSSG values at the bifurcation sites in our study proved that the arterial wall next to the apex was subjected to higher WSS and WSSG values before the generation of aneurysms in models with large bifurcation angles. Elevated WSS or WSSG levels disrupt the normal function of endothelial cells, stimulate gene transcription, and activate ion channels and the consequent reorganization of the cellular cytoskeleton. ${ }^{5,21}$ The more substantial structural changes, which occur after 6 months, appear to involve the upregulation of MMP-2 and -9 , both of which are implicated in aneurysmal disease. ${ }^{25}$

However, this model of the carotid artery bifurcation aneurysm still does not completely represent a true cerebral aneurysm model because compared with extracranial arteries, intracranial arteries have their own specific anatomic and biologic characteristics, which could not be simulated in our model: a well-developed internal elastic lamina; a weakened tunica media and tunica adventitia, or even the absence of the tunica adventitia; reduced elastic fibers; increased proportions of SMCs; and, most important, a CSF environment.

\section{CONCLUSIONS}

We established a new canine model to simulate human arterial bifurcation morphology, including hemodynamic action with accelerated blood velocity and arterial wall degeneration at the bifurcation apex. Aneurysms, confirmed by angiography and histology, formed under persistent low WSS/WSSG and high pressure action in elastase-treated animals, but not in control animals. This canine model has the potential for the identification and study of the genes, proteins, or conduction channels responsible for aneurysm generation, development, and rupture.

\section{REFERENCES}

1. Molyneux A, Kerr R, Stratton, et al. International Subarachnoid Aneurysm Trial (ISAT) of neurosurgical clipping versus endovascular coiling in 2143 patients with ruptured intracranial aneurysms: a randomised trial. Lancet 2002;360:1267-74

2. Hashimoto $\mathrm{T}$, Meng $\mathrm{H}$, Young WL. Intracranial aneurysms: links among inflammation, hemodynamics and vascular remodeling. Neurol Res 2006;28:372-80

3. Canham PB, Finlay HM. Morphometry of medial gaps of human brain artery branches. Stroke 2004;35:1153-57

4. Finlay HM, Whittaker P, Canham PB. Collagen organization in the branching region of human brain arteries. Stroke 1998;29:1595-601

5. Li YS, Haga JH, Chien S. Molecular basis of the effects of shear stress on vascular endothelial cells. J Biomech 2005;38:1949-71

6. Gao L, Hoi Y, Swartz DD, et al. Nascent aneurysm formation at the basilar terminus induced by hemodynamics. Stroke 2008;39: 2085-90

7. Meng H, Wang ZJ, Hoi Y, et al. Complex hemodynamics at the apex of an arterial bifurcation induces vascular remodeling resembling cerebral aneurysm initiation. Stroke 2007;38:1924-31

8. Nuki Y, Tsou TL, Kurihara C, et al. Elastase-induced intracranial aneurysms in hypertensive mice. Hypertension 2009;54:1337-44

9. Aoki T, Nishimura M. The development and the use of experimental animal models to study the underlying mechanisms of CA formation. J Biomed Biotechnol 2011:535921

10. Norman PE, Powell JT. Site specificity of aneurysmal disease. Circulation 2010;121:560-68

11. Sadamasa N, Nozaki K, Hashimoto N. Disruption of gene for inducible nitric oxide synthase reduces progression of cerebral aneurysms. Stroke 2003;34:2980-84

12. Kataoka K, Taneda M, Asai T, et al. Structural fragility and inflammatory response of ruptured cerebral aneurysms: a comparative study between ruptured and unruptured cerebral aneurysms. Stroke 1999;30:1396-401

13. Nixon AM, Gunel M, Sumpio BE. The critical role of hemodynamics in the development of cerebral vascular disease. J Neurosurg 2010;112:1240-53

14. Fischell TA, Virmani R. Intracoronary brachytherapy in the porcine model: a different animal. Circulation 2001;104:2388-90

15. Jung F, Beysang R, Guceve L, et al. Angiography of the cervico-cephalic vessels of the dog: the carotid system [in French]. J Chir (Paris) 1975;109:109-18

16. Oesterle SN, Whitbourn R, Fitzgerald PJ, et al. The stent decade: 1987 to 1997. Am Heart J 1998;136(4 pt 1):578-99

17. Kassam AB, Horowitz M, Chang YF, et al. Altered arterial homeostasis and cerebral aneurysms: a molecular epidemiology study. Neurosurgery 2004;54:1450-60, discussion 1460-62

18. Tulamo R, Frösen J, Hernesniemi J, et al. Inflammatory changes in the aneurysm wall: a review. J Neurointerv Surg 2010;2:120-30

19. Aoki T, Kataoka H, Morimoto M, et al. Macrophage-derived matrix 
metalloproteinase- 2 and -9 promote the progression of cerebral aneurysms in rats. Stroke 2007;38:162-69

20. Watson SP. Platelet activation by extracellular matrix proteins in haemostasis and thrombosis. Curr Pharm Des 2009;15:1358-72

21. Sharma R, Yellowley CE, Civelek M, et al. Intracellular calcium changes in rat aortic smooth muscle cells in response to fluid flow. Ann Biomed Eng 2002;30:371-78

22. Jagadeesan BD, Kadkhodayan Y, Delgado Almandoz JE, et al. Differences in the basilar artery bifurcation angle among patients who present with a ruptured aneurysm at the top of the basilar artery and patients with perimesencephalic subarachnoid hemorrhage: a retrospective cross-sectional study. Neurosurgery 2013;73:2-7
23. Markl M, Wegent F, Zech $\mathrm{T}$, et al. In vivo wall shear stress distribution in the carotid artery: effect of bifurcation geometry, internal carotid artery stenosis, and recanalization therapy. Circ Cardiovasc Imaging 2010;3:647-55

24. Perktold K, Peter RO, Resch M, et al. Pulsatile non-Newtonian blood flow in three-dimensional carotid bifurcation models: a numerical study of flow phenomena under different bifurcation angles. J Biomed Eng 1991;13:507-15

25. Negishi M, Lu D, Zhang YQ, et al. Upregulatory expression of furin and transforming growth factor-beta by fluid shear stress in vascular endothelial cells. Arterioscler Thromb Vasc Biol 2001;21:785-90 